\title{
Étude de cas : Utilisation de lentilles de contact pour la prise en charge des complications suite à une intervention combinant réticulation cornéenne et kératectomie photoréfractive guidée par topographie pour la dégénérescence pellucide marginale
}

D. Yeung, OD, B.Sc., candidate à la maîtrise École d'optométrie et de sciences de la vision Université de Waterloo

\section{Luigina Sorbara}

École d'optométrie et de sciences de la vision, Université de Waterloo

\begin{abstract}
Résumé
Ce cas décrit deux complications à long terme importantes rencontrées par une patiente après le traitement de la dégénérescence pellucide marginale (DPM). Deux ans après avoir subi une kératectomie photoréfractive guidée par topographie (T-PRK) combinée à une procédure de réticulation du collagène cornéen (CXL), la patiente continuait à éprouver des éblouissements et une sécheresse associés à une opacité stromale persistante et à un œil sec. Ces procédures ont causé une insatisfaction avec le résultat final, ce qui a conduit la patiente à utiliser des lentilles de contact pour corriger sa vision. La prise en charge des séquelles oculaires symptomatiques avec des lentilles de contact toriques souples spécialement conçues pour les cornées irrégulières a favorisé sa rééducation visuelle.
\end{abstract}

\section{MOTS CLÉS :}

Kératectomie photoréfractive guidée par topographie (T-PRK), réticulation du collagène cornéen $(\mathrm{CXL})$, dégénérescence pellucide marginale (DPM), opacité stromale, œil sec 
L

a dégénérescence pellucide marginale (DPM) est une pathologie ectasique non inflammatoire progressive impliquant la région périphérique inférieure de la cornée ${ }^{1-3}$. Dans cette affection, un amincissement en forme de croissant s'étendant des positions 4 heures à 8 heures se produit dans la cornée périphérique. Alors que le degré d'astigmatisme irrégulier dépend de la gravité de la maladie, la distorsion visuelle secondaire à la DPM est généralement asymptomatique dans les premiers stades, car la zone d'amincissement n'implique pas l'axe visuel. C'est pourquoi la topographie cornéenne est la méthode diagnostique de référence pour cette affection ${ }^{3}$. La pratique courante dans la prise en charge des DPM comprend des lunettes, des lentilles de contact et des interventions chirurgicales telles que la kératoplastie lamellaire ou pénétrante ${ }^{1,4,5}$. Récemment, une kératectomie photoréfractive guidée par topographie simultanée (T-PRK) et des procédures de réticulation du collagène cornéen (CXL) ont été utilisées pour traiter la DPM et d'autres causes de kératectasie dans le but de ralentir la progression de la maladie, de minimiser l'irrégularité cornéenne et de réduire la nécessité d'une transplantation cornéenne ${ }^{6-10}$.

La T-PRK implique une ablation du tissu stromal cornéen «sur mesure » au laser Excimer guidé par l'aberrométrie du front d'onde, la tomographie et la topographie ${ }^{11-13}$. En aplatissant l'apex cornéen ou le tissu élevé et raidi adjacent aux zones ectasiques, cette procédure normalise la cornée en réduisant l'astigmatisme et l'irrégularité de la surface de la cornée $e^{6,11,13}$. Le CXL utilise l'effet photosensibilisant de la riboflavine et les rayons ultraviolets A (UVA) pour induire la polymérisation des fibres de collagène dans les 200 à 300 microns antérieurs du stroma cornéen $^{14-18}$. Le CXL augmente efficacement la rigidité cornéenne et la force biomécanique par trois fois ${ }^{8,1711}$. Il entraîne également un aplatissement simultané de la zone la plus abrupte et une accentuation de la courbure des zones adjacentes autour du cône, ce qui diminue l'asymétrie cornéenne et l'aberration sphérique ${ }^{19}$. La combinaison de ces procédures, le CXL étant réalisé immédiatement après la T-PRK, est bénéfique dans la gestion de l'ectasie cornéenne ${ }^{810}$. Il a été démontré que le temps d'intervention peut être réduit si ces procédures sont effectuées simultanément ${ }^{20}$. Ceci permet également à la riboflavine d'atteindre les couches profondes du tissu stromal et d'y induire la réticulation du collagène $e^{20}$. De plus, ceci minimise le risque de cicatrices stromales superficielles résultant de la PRK en diminuant l'activité des fibroblastes après l'ablation des kératocytes6. L'inversion de l'ordre dans lequel les procédures sont effectuées aboutirait soit à l'élimination du tissu cornéen réticulé durci, soit à une réduction du taux d'ablation des tissus réticulés possédant des propriétés biomécaniques modifiées, aucun de ces résultats n'étant bénéfique ${ }^{8,10,20}$.

L'efficacité de la T-PRK et du CXL effectués simultanément pour suspendre temporairement la progression de l'ectasie et améliorer les acuités visuelles non corrigées et corrigées dans les cas de DPM a été bien documentée ${ }^{8,10}$. Cependant, il y a peu d'information disponible en ce qui concerne les complications qui peuvent survenir suite à ces procédures. Ce rapport de cas décrit deux de ces complications (opacité permanente du stroma cornéen et œil sec) et leur prise en charge.

\section{RAPPORT DE CAS}

Une femme de 41 ans de race blanche s'est présentée à la clinique de lentilles cornéennes de l'École d'optométrie et de sciences de la vision de l'Université de Waterloo pour une consultation sur les lentilles de contact spécialisées. Ses principales préoccupations visuelles comprenaient une sensibilité à la lumière et un problème d'éblouissement importants, qui étaient tous deux suffisamment graves pour avoir une incidence sur ses activités quotidiennes. D'après les renseignements qui figuraient à son dossier oculaire, une DPM bilatérale avait été diagnostiquée cinq ans auparavant lors d'un rendez-vous de consultation en vue d'une chirurgie réfractive, en 2010. Peu après le diagnostic, elle a subi une intervention combinant une T-PRK suivie d'un CXL dans les deux yeux. La patiente attribue ses symptômes visuels débilitants et son problème d'œil sec à ces procédures. Dans l'ensemble, sa santé générale était bonne, exception faite de migraines occasionnelles. Un examen médical antérieur avait exclu le syndrome de Sjögren. Les médicaments qu'elle prenait au moment de la consultation comprenaient la cyclosporine (Restasis'), la duloxétine (Cymbalta), le Lorazépam et la zopiclone (Imovane").

Ses antécédents médicaux oculaires comptaient une expérience antérieure avec des lentilles souples jetables perméables au gaz qui n'avait pas été couronnée de succès, principalement en raison de ses symptômes sévères de sécheresse, qui ont finalement conduit à une intolérance aux lentilles de contact. On lui avait déjà prescrit une série de traitements standard pour les yeux secs, dont les soins d'hygiène de la paupière et les compresses chaudes, l'application fréquente de larmes artificielles avec et sans composants lipidiques, un traitement d'un mois de lotéprednol topique, deux ans de cyclosporine A topique et une occlusion du méat inférieur bilatérale. Aucune de ces thérapies antérieures n'avait rétabli le confort visuel de la patiente à un niveau qui lui permettait d'exercer ses activités quotidiennes. 
Au cours de l'examen oculaire, la réfraction était de -0,75/-2,50 x $148\left(6 / 9^{-2}\right)$ pour l'œil droit et -0,25/-2,25 X 057 $\left(6 / 9^{+2}\right)$ pour l'œil gauche. Toutes les analyses de départ étaient normales. Ses pupilles étaient de taille égale à environ 8,0 mm dans des conditions scotopiques et 5,0 $\mathrm{mm}$ dans des conditions photopiques.

L'examen du segment antérieur au biomicroscope a révélé une opacité stromale centrale dans les deux yeux, avec une prise fluo significative de l'épithélium de la cornée inférieure, classé à 2+. On a également observé une opacité stromale centrale bilatérale à l'aide d'un système d'imagerie par tomographie à cohérence optique (OCT) du segment antérieur (Visante ${ }^{\mathrm{T}}$, Zeiss, Jena, Allemagne) (la figure 1A montre l'œil droit et la figure 1B montre l'œil gauche). En outre, la ligne de démarcation résultant de la procédure CXL pouvait être observée et l'opacité cornéenne s'étendait à travers le stroma antérieur et postérieur. Un dysfonctionnement léger à modéré de la glande de Meibomian a été observé dans les deux yeux. Le Tear break-up time (TBUT) était de 4 secondes pour l'œil droit et de 5 secondes pour l'œil gauche. Un test du fil de coton au phénol rouge (phenol red thread test, PRTT) a donné des résultats de $22 \mathrm{~mm}$ dans l'œil droit et de $18 \mathrm{~mm}$ dans l'œil gauche, ce qui a exclu l'œil sec avec insuffisance lacrymale.

Figure $1 A$

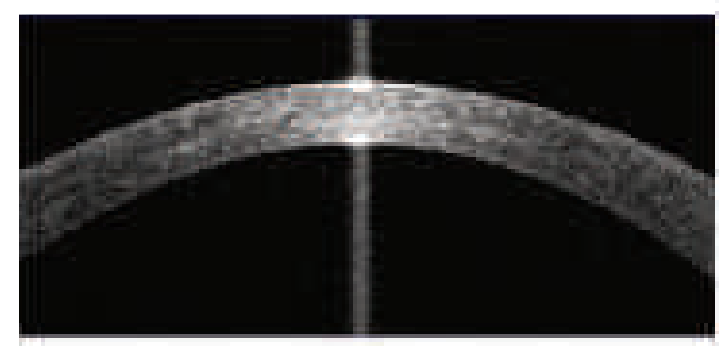

Figure 1B

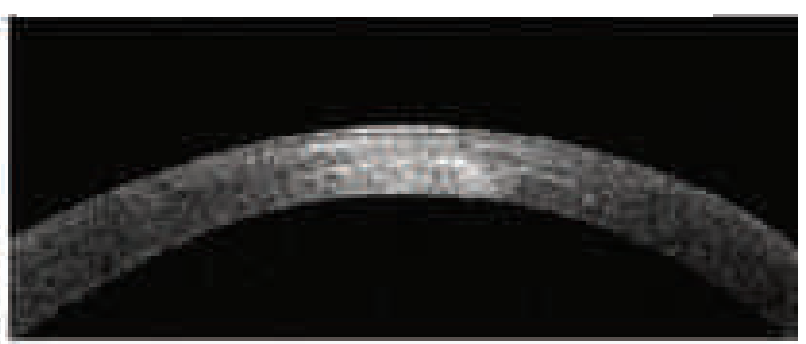

Les topographies cornéennes obtenues avec un système Pentacam ${ }^{\text {TM }}$ (Oculus, Wetzlar, Allemagne) ont révélé des valeurs de kératométrie simulées de 38,4D@16,8 \% /40,0D dans l'œil droit et de 38,8D@67,5\%39,6D dans l'œil gauche (figure 2).

Figure $2 A$

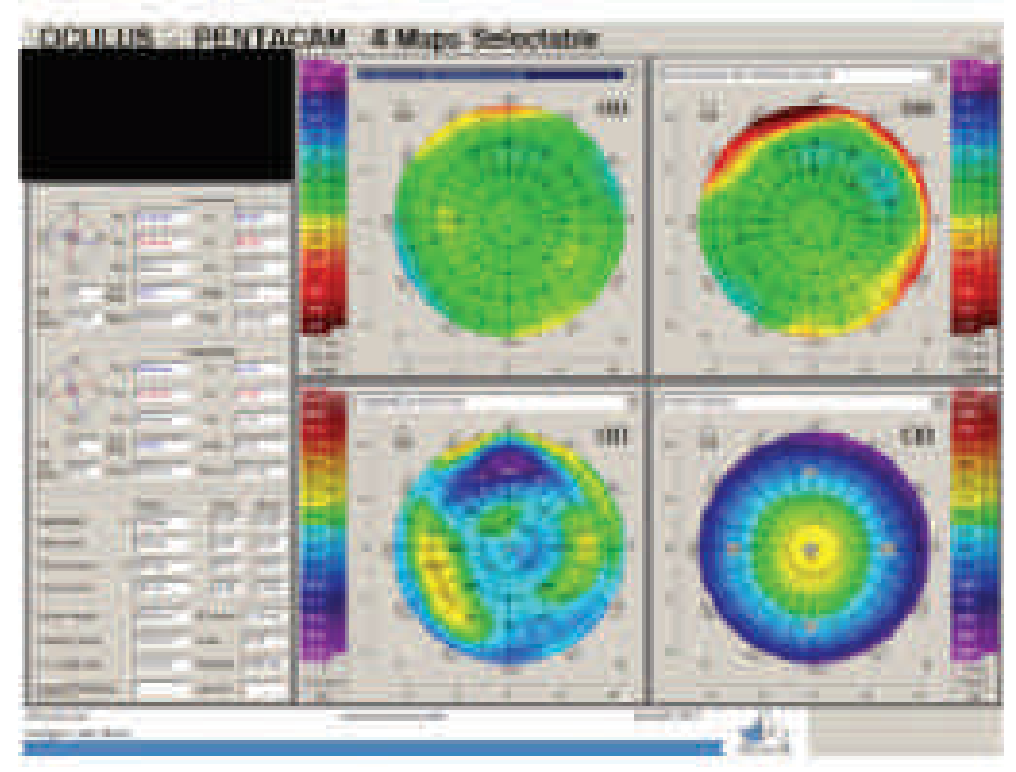




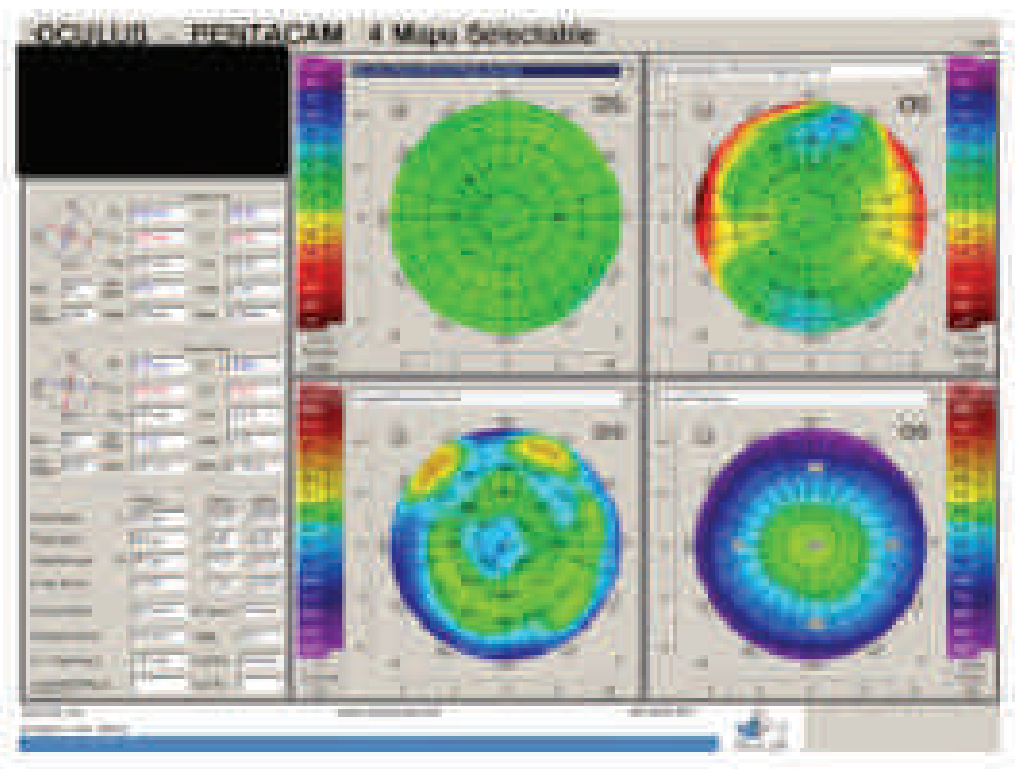

D’après les renseignements consignés dans son dossier médical au moment de la chirurgie réfractive, ses lectures kératométriques simulées de base étaient de 43,00D@097\%/45,00 dans l'œil droit et de 45,00D@015\%/46,00 dans l'œil gauche. La pachymétrie mesurée au point le plus mince était de $564 \mu \mathrm{m}$ dans l'œil droit et allait de $247 \mu \mathrm{m}$ à $555 \mu \mathrm{m}$ dans l'œil gauche avec une grande variabilité entre les mesures. Un dysfonctionnement léger à modéré de la glande de Meibomian sans anomalies cornéennes a été noté lors d'un examen du segment antérieur.

Nous avons conclu que le patient présentait des symptômes visuels secondaires à l'opacité cornéenne survenue suite à la T-PRK et au CXL, ainsi qu'aux symptômes de kératoconjonctivite sèche qui se sont aggravés après la procédure. Suite à des discussions approfondies sur les options de prise en charge avec la patiente, nous avons décidé qu'elle pourrait bénéficier de lentilles de contact spécialisées. Les premières lentilles de contact spécialisées prises en considération pour cette patiente étaient des lentilles sclérales. Pour faciliter l'adaptation, nous avons choisi une lentille sclérale avec un diamètre global plus petit et une épaisseur centrale plus mince. Selon le guide d'ajustement Rose K2 $\mathrm{XL}^{\mathrm{TM} 21}$ (Blanchard Laboratories, Sherbrooke, Québec), nous avons sélectionné les lentilles diagnostiques suivantes :

OD : Rose K2 XL/8,00/14,6/plan/Périphérie standard

OS : Rose K2 XL/8,10/14,6/plan/Périphérie standard

Les lentilles ont été appliquées, avec un temps de stabilisation de 30 minutes. Leurs caractéristiques d'ajustement ont ensuite été examinées. Les deux lentilles ont démontré un dégagement apical excessif et un dégagement minimal du limbe. Les lentilles sont bien centrées et offrent une bonne couverture. Une légère élévation du bord a été notée dans les deux yeux à 3 et 9 heures. La patiente a également signalé une sensation de corps étranger due à la lentille de légère à modérée chez les deux yeux. L'ajustement des lentilles était jugé trop cambré au centre. Sur la base de l'évaluation et de la surréfraction des lentilles d'essai, nous avons placé une commande finale avec les paramètres suivants :

OD : Rose K2 XL/8,00/14,8/-4,00/Diminution de l'élévation du bord nº 1

OS : Rose K2 XL/8,30/14,8/-2,75/Diminution de l'élévation du bord nº 1 


\section{Première visite de suivi}

Au rendez-vous de délivrance des lentilles, l'ajustement des lentilles a été jugé approprié, avec un dégagement apical adéquat. La patiente a continué de signaler une sensation de corps étranger légère à modérée. Elle a été avisée que cette sensation initiale de lentille est normale pour un porteur de lentille sclérale novice, et on lui a recommandé d'augmenter lentement le temps de port pour faciliter le processus d'adaptation, en commençant par deux à trois heures par jour jusqu'à son prochain rendez-vous de suivi. On lui a également expliqué comment insérer et enlever les lentilles sclérales correctement avec des techniques de manipulation appropriées. Les lentilles ont été délivrées à la patiente avec une trousse de départ de la solution Daily Boston Simplus " (Bausch et Lomb, Rochester, NY).

\section{Deuxième visite de suivi}

Au cours de la visite de suivi de deux semaines, l'évaluation des lentilles à l'aide d'un biomicroscope a confirmé que les lentilles étaient bien centrées avec un dégagement apical approprié d'environ 75 microns, conformément au manuel d'ajustement Rose $\mathrm{K} 2 \mathrm{XL}^{21}$. Une compression légère, mais tolérable du limbe a été notée dans la région supérieure et inférieure des deux yeux. La surréfraction sphérocylindrique était de +1,00-2,00x105 (6/7,5) dans l'œil droit et de $+0,25-1,00 \times 090\left(6 / 7,5^{-3}\right)$ dans l'œil gauche. Cependant, bien que la patiente ait signalé une réduction de ses symptômes de sécheresse avec le port des lentilles, elle était insatisfaite du confort général des lentilles de contact et notait que la sensation de corps étranger due à la présence de la lentille était plus forte dans l'œil gauche que dans l'œil droit. En fin de compte, nous avons conclu que la patiente devrait cesser l'utilisation de lentilles sclérales en raison d'un confort insuffisant et d'une mauvaise adaptation à ce type de lentille.

La procédure d'ajustement a été répétée avec des Kerasoft IC (Bausch + Lomb), une lentille kératocônique souple « sur mesure », comme alternative pour la gestion de ses symptômes visuels. Nous avons commandé des lentilles avec les paramètres suivants :

OD : Kerasoft ${ }^{\circ}$ IC/8,20/14,5/-0,50 -0,75 x 052/Périphérie aplatie nº 1

OS : Kerasoft" IC/8,60/14,5/-1,25 -1,00 x 075/Périphérie standard

\section{Troisième visite de suivi}

Lors du rendez-vous de suivi de deux semaines pour les verres Kerasoft ${ }^{\circ} \mathrm{IC}$, la patiente s'est montrée satisfaite et a rapporté une amélioration subjective de la qualité de sa vision sans fatigue oculaire avec le port des lentilles. Elle a déclaré un bon confort général avec les lentilles. Cependant, elle ne pouvait porter ses lentilles que deux heures par jour, après quoi sa vision devenait voilée. Elle utilisait la solution polyvalente Biotrue ${ }^{\mathrm{rnt}}$ (Bausch + Lomb) pour nettoyer et entreposer les lentilles chaque soir. La patiente a obtenu des acuités visuelles de 6/7,5 dans l'œil droit et 6/9 + dans l'œil gauche sans amélioration significative de la vision avec une surréfraction sphérique. Dans une évaluation des lentilles, les deux lentilles étaient bien centrées avec un mouvement adéquat pour le regard fixe primaire ou le regard vers le haut. La rotation était stable à 5 degrés dans le sens inverse des aiguilles d'une montre dans l'œil droit et à 12 degrés dans le sens inverse des aiguilles d'une montre dans l'œil gauche. Une quantité modérée de dépôts lipidiques a été trouvée sur ses lentilles. À la fin du rendez-vous, nous avons décidé de continuer avec ces lentilles sans changer les paramètres, mais de passer de son système de soins à Peroxiclear ${ }^{\text {rin }}$ (Bausch + Lomb). Des gouttes oculaires Blink et Clean ${ }^{\text {TI" }}$ complètes (Abbott Medical Optics, Abbott Park, IL) ont été recommandées au besoin pendant le port des lentilles de contact.

\section{Quatrième visite de suivi}

La patiente a signalé de bons résultats avec les verres Kerasoft ${ }^{\circ}$ IC. Son temps d'utilisation était passé à sept heures par jour, trois à quatre jours par semaine avec le changement de son système de soins et l'utilisation de gouttes pour les yeux Complete Blink and Clean ${ }^{\text {TII }}$ (Abbott Medical Optics) quatre fois par jour. La patiente a continué à éprouver un éblouissement subjectif avec les lentilles, probablement en raison de la grande taille de ses pupilles en conditions photopique et scotopique et de l'opacité cornéenne importante survenue suite aux procédures de T-PRK et de CXL simultanées. Les lentilles kératocôniques souples personnalisées ont rétabli avec succès sa vision fonctionnelle pour les activités quotidiennes normales. Elle sera suivie tous les trois mois.

\section{DISCUSSION}

La dégénérescence pellucide marginale (DPM) est une kératectasie non inflammatoire progressive qui cause un amincissement de la région inférieure de la cornée et un astigmatisme irrégulier ${ }^{1-3}$. L'utilisation de lentilles de contact sclérocornéennes perméables aux gaz peut corriger l'astigmatisme irrégulier qui est induit à mesure que la maladie progresse et que la cornée s'amincit. Il a été démontré que la T-PRK et le CXL simultanés peuvent augmenter l'efficacité du CXL 
en permettant une meilleure pénétration de la solution de riboflavine utilisée avec le CXL, en raison de l'absence de la couche de Bowman et de la résection partielle du stroma ${ }^{20}$. Des procédures simultanées peuvent aussi réduire l'opacité sous-épithéliale qui suit généralement la $\mathrm{PRK}^{6}$. La technique combinant la T-PRK et le CXL peut être une option efficace pour la prise en charge de la DPM et peut améliorer le pronostic en réduisant le degré et l'irrégularité de l'astigmatisme cornéen. Cependant, il y a peu d'information sur les complications et la satisfaction des patients après l'intervention. Cette étude de cas a présenté une patiente âgée de 41 ans qui présentait une opacité cornéenne permanente cliniquement significative et des symptômes de sécheresse oculaire après avoir subi une T-PRK et un CXL combinés.

L’opacité cornéenne permanente suite au CXL est définie comme une opacité stromale qui persiste pendant plus de six mois après le CXL et a été documentée dans $5 \%$ à $8,6 \%$ des cas ${ }^{22,23}$. L'opacité cornéenne qui suit un CXL s'étend généralement à travers les 300 microns antérieurs du stroma et peut être différenciée des séquelles de la PRK parce que celles ci sont sous-épithéliales et beaucoup moins profondes ${ }^{15,18,24,25}$. Par ailleurs, cette complication à long terme du CXL est différente de l'opacité centrale lacunaire transitoire de type nid d'abeilles qui se produit dans les 300 microns antérieurs du stroma cornéen, présente dans tous les yeux à 1 mois après le CXL ${ }^{26,27}$. La présence d'une opacité transitoire, qui ne s'étend qu'à travers la profondeur du stroma cornéen affecté par le CXL est un signe que la procédure de CXL est réussie. Avec l'utilisation d'un traitement stéroïdien topique sans conservateur, qui est généralement prescrit pendant 1 à 3 mois suite à l'intervention, la perte de transparence de la cornée diminue sur une période de 6 mois à 1 an après le CXL, ce qui coïncide avec l'achèvement du repeuplement kératocytaire ${ }^{22,27}$. D'autre part, une opacité stromale permanente reflète les changements dans les protéines cristallines et les interconnexions lamellaires induits par l'interaction de la riboflavine et des rayons ultraviolets $\mathrm{A}^{28,29}$. Bien qu'il n'ait pas été montré que cette opacité résistante aux stéroïdes entraîne une réduction de l'acuité visuelle à fort contraste, elle peut augmenter la rétrodiffusion de la lumière qui est réfléchie à l'intérieur de la cornée ${ }^{22,29-31}$. Il est donc possible qu'elle ait conduit à la dégradation de la qualité visuelle et aux symptômes débilitants d'éblouissement et de distorsion visuelle dus à ces aberrations qu'a ressentis la patiente dans ce cas. Raiskup et coll. ont noté un risque plus élevé d'opacité stromale permanente après la CXL dans les cas de kératectasie sévère avec des valeurs kératométriques préopératoires plus élevées ${ }^{23}$. Ces cas présentaient probablement des anomalies stromales intrinsèques associées aux kératectasies telles que des microstries et des noyaux de kératocytes hyperactivés dans le tissu stromal, et la présence de stries de Vogt avant la procédure de $\mathrm{CXL}^{23}$.

En ce qui concerne les traitements possibles, il n'y a eu aucune étude à ce jour sur les avantages potentiels de la mitomycine $\mathrm{C}$, un agent antiprolifératif dont on a montré qu'il réduit le risque d'opacité cornéenne suite à une PRK, sur la réduction de l'opacité stromale qui survient après un CXL32. L'utilisation de la mitomycine C chez les patients atteints de kératectasie peut être potentiellement contre-indiquée en raison des propriétés cytotoxiques de la mitomycine $\mathrm{C}$ et le rôle qu'elle joue dans l'induction de l'apoptose des kératocytes peut augmenter le risque d'accélérer l'amincissement cornéen.

Notre patiente a présenté une opacité cornéenne importante à travers toute l'épaisseur du stroma dans les deux yeux, comme montré dans les lectures d'OCT du segment antérieur à la figure 1. Les lectures montrent également une ligne de démarcation à travers le stroma qui indique l'étendue du stroma antérieur affecté par le CXL ${ }^{15,18,2527,30,33}$. Cette présentation de l'opacité stromale qui s'étend dans le stroma postérieur est atypique de l'opacité stromale qui apparaît après la procédure de CXL. Güell et coll. ont rapporté le développement d'une opacité stromale postérieure 5 mois après une intervention de T-PRK et de CXL simultanés chez un patient de 22 ans avec un kératocône fruste ${ }^{34}$. Dans une autre série de cas, des structures linéaires hyperréfléchissantes ont été observées dans 13 des 28 yeux qui avaient subi une T-PRK et un CXL simultanés pour la prise en charge du kératocône ${ }^{35}$. Cependant, les auteurs n'ont trouvé aucune corrélation entre la prévalence de l'opacité stromale postérieure et l'épaisseur préopératoire de la cornée du patient ou la profondeur de l'ablation.

La sécheresse qui survient après une intervention kératoréfractive est l'une des principales causes de l'insatisfaction des patients suite à l'intervention ${ }^{36}$. L'interruption du système de rétroaction neurale due à une réduction de la sensibilité cornéenne a été proposée comme principale cause de l'œil sec après une intervention kératoréfractive ${ }^{37,38}$. Dans le cas de procédures de T-PRK et de CXL combinées, on élimine l'épithélium en utilisant de l'alcool topique, un grattage mécanique, une brosse rotative ou un laser, suivi d'une irradiation des 300 microns antérieurs du tissu stromal avec des $\mathrm{UVA}^{20,39,40}$. Cette procédure entraîne la rupture du plexus nerveux sous-épithélial et antérieur-médian du stroma, ce qui entraîne une diminution transitoire de la production des glandes lacrymales dans les six premiers mois qui suivent l'intervention ${ }^{29,38,39}$. La régénération du plexus sous-épithélial à partir de la zone non irritée environnante commence à la fin du premier mois, tandis que l'extension du plexus nerveux stromal plus profond dans le stroma antérieur peut être observée dès le deuxième ou le troisième mois ${ }^{29}$. Des études par microscopie confocale ont confirmé que le nombre de fibres nerveuses est essentiellement rétabli aux valeurs de base préopératoires six mois après la procédure, alors que le nombre d'interconnexions entre les fibres neurales continue à augmenter jusqu'à deux ans après la procé- 
dure ${ }^{29,41}$. La sensibilité cornéenne retourne à sa ligne de base préopératoire dans l'année qui suit la procédure, et la sécrétion de larme de base et la stabilité du film lacrymal sont habituellement restaurées simultanément ${ }^{29,42,43}$.

Chez cette patiente, on a mesuré une PRTT supérieure à $15 \mathrm{~mm}$ dans les deux yeux avec un TBUT réduit, ce qui suggère la présence d'autres facteurs pouvant avoir contribué aux signes et symptômes de sécheresse. Tout d'abord, la cornée prend une topographie oblate après une chirurgie kératoréfractive. L’irrégularité de la surface de la cornée, confirmée par la présence d'un cylindre cornéen oblique, peut constituer un obstacle à la répartition régulière du film lacrymal lors du clignement de l'œil. Deuxièmement, l'écart entre les symptômes signalés et les signes de sécheresse peut s'expliquer par une excitabilité altérée dans les nerfs régénérés causée par le processus inflammatoire induit après une lésion du nerf cornéen ${ }^{44}$. Troisièmement, on sait que l'utilisation à long terme d'anxiolytiques a un effet anti-cholinergique qui entraîne une diminution de la production aqueuse et des symptômes d'œil sec ${ }^{45,46}$. Enfin, la diminution naturelle de la population de cellules caliciformes avec l'âge peut contribuer à compromettre la couche de mucine, ce qui se traduit par l'instabilité du film lacrymal ${ }^{39,47}$.

Dans cette étude de cas, une patiente de 41 ans a subi une intervention combinant une T-PRK et un CXL peu de temps après un diagnostic de DPM. Si l'on prend en considération le fait que la patiente était visuellement asymptomatique avant son diagnostic, sa qualité de vie a sans doute diminué après l'opération en raison de ses symptômes débilitants d'éblouissement, de sensibilité à la lumière et de sécheresse. L'opacité cornéenne bilatérale et permanente qui s'est développée suite au CXL et l'augmentation de la réflectivité interne (diffusion de la lumière) dans le tissu stromal qui en a résulté sont responsables de ses symptômes visuels. Ces symptômes sont probablement exacerbés par ses pupilles plus grandes que la moyenne, soit environ 8,0 $\mathrm{mm}$ dans des conditions scotopiques et 5,0 mm dans des conditions photopiques. Les procédures de T-PRK et de CXL ont exacerbé ses symptômes de sécheresse oculaire; elle était déjà à haut risque en raison de son âge, d'une histoire préopératoire de dysfonctionnement de la glande de Meibomian, de l'utilisation d'un contraceptif oral, et de son utilisation actuelle de médicaments antidépresseurs et anxiolytiques. Bien que cette patiente soit maintenant prise en charge avec des lentilles toriques spécialisées pour sa cornée irrégulière, un dépistage préopératoire soigneux accordant une attention particulière à la taille des pupilles et aux antécédents médicaux oculaires peut aider à identifier les patients qui sont plus à risque de développer des résultats indésirables.

\section{CONCLUSIONS}

La T-PRK et le CXL simultanés peuvent améliorer efficacement les acuités visuelles corrigées et non corrigées tout en ralentissant la progression de la DPM et d'autres kératectasies ${ }^{8,10}$. Cette combinaison de procédures peut retarder efficacement la nécessité d'une prise en charge plus invasive comme la kératoplastie pénétrante et l'implantation de segments annulaires intrastromiques ${ }^{17}$. Ce rapport de cas a examiné deux complications à long terme importantes qui surviennent suite à ces procédures (éblouissement associé à l'opacité stromale persistante et sécheresse chronique), qui ont entraîné l'insatisfaction de la patiente avec le résultat. Un examen plus attentif des critères d'admissibilité pour ces procédures pourrait accroître les avantages relatifs du ralentissement de la progression de la DPM tout en diminuant les risques relatifs de complications. 
RÉFÉRENCES :

1. Sridhar MS, Mahesh S, Bansal AK, et al. Pellucid marginal corneal degeneration. Ophthalmology 2004;111:1102-7. doi:10.1016/j. ophtha.2003.09.035.

2. Krachmer JH. Pellucid marginal corneal degeneration. Arch Ophthalmol 1978;96(7):1217-21.

3. Jinabhai A, Radhakrishnan H, O'Donnell C. Pellucid corneal marginal degeneration: A review. Contact Lens Anterior Eye 2011;34(2):56-63. doi:10.1016/j.clae.2010.11.007.

4. Biswas S, Brahma A, Tromans C, et al. Management of pellucid marginal corneal degeneration. Eye 2000;14(4):629-34. doi:10.1097/01.ico.0000153555.82278.5b.

5. Varley GA, Macsai MS, Krachmer JH. The results of penetrating keratoplasty for pellucid marginal corneal degeneration. Am J Ophthalmol 1990;110(2):149-52.

6. Kanellopoulos AJ. Comparison of sequential vs same-day simultaneous collagen cross-linking and topography-guided PRK for treatment of keratoconus. J Refract Surg 2009;25(9):S812-8. doi:1 0.3928/1081597X-20090813-10.

7. Wollensak G, Spoerl E, Seiler T. Stress-strain measurements of human and porcine corneas after riboflavin-ultraviolet-Ainduced cross-linking. J Cataract Refract Surg 2003;29:1780-5. doi:10.1016/S0886-3350(03)00407-3.

8. Kymionis GD, Karavitaki AE, Kounis GA, et al. Management of pellucid marginal corneal degeneration with simultaneous customized photorefractive keratectomy and collagen crosslinking. J Cataract Refract Surg 2009;35(7):1298-301. doi:10.1016/j. jcrs.2009.03.025.

9. Stojanovic A, Zhang J, Chen X, et al. Topography-guided transepithelial surface ablation followed by corneal collagen cross-linking performed in a single combined procedure for the treatment of keratoconus and pellucid marginal degeneration. J Refract Surg 2010;26(2):145-52. doi:10.3928/108159 7X-20100121-10.

10. Kymionis GD, Kontadakis GA, Kounis GA, et al. Simultaneous topography-guided PRK followed by corneal collagen cross-linking for keratoconus. J Refract Surg 2009;25(September):807-12. doi:10.3928/1081597X-20090813-09.

11. Kanellopoulos AJ. Managing highly distorted corneas with topography-guided treatment. ISRS/AAO 2007 Subspecialty Day/Refractive Surg Syllabus Sect II Ablation Strategies 2007:135 .

12. Cennamo G, Intravaja A, Boccuzzi D, et al. Treatment of keratoconus by topography-guided customized photorefractive keratectomy: Two-year follow-up study. J Refract Surg 2008;24(2):145-9.

13. Lin DTC, Holland SR, Rocha KM, et al. Method for optimizing topography-guided ablation of highly aberrated eyes with the ALLEGRETTO WAVE excimer laser. J Refract Surg 2008;24(4):S439-45.

14. Caporossi A, Mazzotta C, Baiocchi S, et al. Long-term results of riboflavin ultraviolet A corneal collagen cross-linking for keratoconus in Italy: The Siena Eye Cross Study. Am J Ophthalmol 2010;149(4):585-93. doi:10.1016/j.ajo.2009.10.021.

15. Dhawan S, Rao K, Natrajan S. Complications of corneal collagen cross-linking. J Ophthalmol 2011;2011:869015. doi:10.1155/2011/869015.

16. Spadea L. Corneal collagen cross-linking with riboflavin and UVA irradiation in pellucid marginal degeneration. J Refract Surg 2010;26(5):375-7. doi:10.3928/1081597X-20100114-03.

17. Wollensak G, Spoerl E, Seiler T. Riboflavin/ultraviolet-A-induced collagen crosslinking for the treatment of keratoconus. Am J Ophthalmol 2003;135:620-7. doi:10.1016/S0002-9394(02)02220-1.

18. Dahl BJ, Spotts E, Truong JQ. Corneal collagen cross-linking: An introduction and literature review. Optometry 2012;83(1):33-42. doi:10.1016/j.optm.2011.09.011.

19. Vinciguerra P, Albè E, Trazza S, et al. Refractive, topographic, tomographic, and aberrometric analysis of keratoconic eyes undergoing corneal cross-linking. Ophthalmology 2009;116(3):369 78. doi:10.1016/j.ophtha.2008.09.048.

20. Kanellopoulos AJ, Binder PS. Management of corneal ectasia after LASIK with combined, same-day, topography-guided partial transepithelial PRK and collagen cross-linking: the athens protocol. J Refract Surg 2011;27(5):323-31. doi:10.3928/108159
7X-20101105-01.

21. Blanchard Contact Lens Inc. Rose K2 XL Semi-scleral lens Fitting Guide. Canada (Toronto, ON)

22. Mazzotta C, Balestrazzi A, Traversi C, et al. Treatment of progressive keratoconus by riboflavin-UVA-induced cross-linking of corneal collagen: ultrastructural analysis by Heidelberg Retinal Tomograph II in vivo confocal microscopy in humans. Cornea 2007;26:390-7. doi:10.1097/ICO.0b013e318030df5a.

23. Raiskup F, Hoyer A, Spoerl E. Permanent corneal haze after riboflavin-UVA-induced cross-linking in keratoconus. J Refract Surg 2009;25(9):S824-8. doi:10.3928/1081597X-20090813-12.

24. Eliacik M, Bayramlar H, Erdur SK, et al. Anterior segment optical coherence tomography evaluation of corneal epithelium healing time after 2 different surface ablation methods. Saudi Med J 2015;36(1):67-72. doi:10.15537/smj.2015.1.9983.

25. Meek KM, Hayes S. Corneal cross-linking - a review. Ophthalmic Physiol Opt 2013;33(2):78-93. doi:10.1111/opo.12032.

26. Wollensak G, Herbst H. Significance of the lacunar hydration pattern after corneal cross linking. Cornea 2010;29:899-903. doi:10.1097/ICO.0b013e3181ca3293.

27. Koller T, Mrochen M, Seiler T. Complication and failure rates after corneal crosslinking. J Cataract Refract Surg 2009;35(8):1358-62. doi:10.1016/j.jcrs.2009.03.035.

28. Jester J V, Moller-Pedersen T, Huang J, et al. The cellular basis of corneal transparency: Evidence for "corneal crystallins". J Cell Sci 1999;112 (Pt 5):613-22.

29. Bovone C, Sparano MC, Balestrazzi A, et al. Corneal healing after riboflavin ultraviolet-A collagen cross-linking determined by confocal laser scanning microscopy in vivo: Early and late modifications. Am J Ophthalmol 2008;146:527-34. doi:10.1016/j. ajo.2008.05.042.

30. Greenstein SA, Fry KL, Bhatt J, et al. Natural history of corneal haze after collagen crosslinking for keratoconus and corneal ectasia: Scheimpflug and biomicroscopic analysis. J Cataract Refract Surg 2010;36(12):2105-14. doi:10.1016/j.jcrs.2010.06.067.

31. Connon CJ, Marshall J, Patmore AL, et al. Persistent haze and disorganization of anterior stromal collagen appear unrelated following phototherapeutic keratectomy. J Refract Surg 2002;19(3):323-32.

32. Teus MA, de Benito-Llopis L, Alió JL. Mitomycin C in corneal refractive surgery. Surv Ophthalmol 2009;54(4):487-502. doi:10.1016/j.survophthal.2009.04.002.

33. Kanellopoulos AJ, Binder PS. Collagen cross-linking (CCL) with sequential topography-guided PRK: A temporizing alternative for keratoconus to penetrating keratoplasty. Cornea 2007;26(7):891-5. doi:10.1097/ICO.0b013e318074e424.

34. Güell JL, Verdaguer P, Elies D, et al. Late onset of a persistent, deep stromal scarring after PRK and corneal cross-linking in a patient with forme fruste keratoconus. J Refract Surg 2014;30(4):286-8. doi:10.3928/1081597X-20140320-09.

35. Kymionis GD, Portaliou DM, Diakonis VF, et al. Posterior linear stromal haze formation after simultaneous photorefractive keratectomy followed by corneal collagen cross-linking. Invest Ophthalmol Vis Sci 2010;51(10):5030-3. doi:10.1167/iovs.09-5105.

36. Jabbur NS, Sakatani K, O'Brien TP. Survey of complications and recommendations for management in dissatisfied patients seeking a consultation after refractive surgery. J Cataract Refract Surg 2004;30(9):1867-74. doi:10.1016/j.jcrs.2004.01.020.

37. Quinto GG, Camacho W, Behrens A. Postrefractive surgery dry eye. Curr Opin Ophthalmol 2008;19(4):335-41.

38. Ang RT, Dartt DA, Tsubota K. Dry eye after refractive surgery. Curr Opin Ophthalmol 2001;12:318-22. doi:10.1097/00055735200108000-00013.

39. Dartt DA. Dysfunctional neural regulation of lacrimal gland secretion and its role in the pathogenesis of dry eye syndromes. Ocul Surf 2004;2(2):76-91. doi:10.1016/S1542-0124(12)70146-5.

40. Kymionis GD, Mikropoulos DG, Portaliou DM, et al. An overview of corneal collagen cross-linking (CXL). Adv Ther 2013;30(10):858-69. doi:10.1007/s12325-013-0065-9.

41. Murakami Y, Manche EE. Prospective, randomized comparison of self-reported postoperative dry eye and visual fluctuation in LASIK and photorefractive keratectomy. Ophthalmology 2012;119(11):2220-4. doi:10.1016/j.ophtha.2012.06.013. 
42. Kontadakis GA, Kymionis GD, Kankariya VP, et al. Effect of corneal collagen cross-linking on corneal innervation, corneal sensitivity, and tear function of patients with keratoconus. Ophthalmology 2013;120:917-22. doi:10.1016/j.ophtha.2012.10.012.

43. Dash SK. Corneal sensitivity is reduced and relates to the severity of neuropathy in patients with diabetes: response to Tavakoli et al. Diabetes Care 2007;30:e142; author reply e143. doi:10.2337/ dc07-1786.

44. Cruzat A, Pavon-Langston D, Hamrah P, et al. In vivo confocal microscopy of corneal nerves: Analysis and clinical correlation. Semin Ophthalmol 2010;25(5-6):171-7. doi:10.3109/08820538.201 0.518133 .

45. Jaanus SD. Ocular side effects of selected systemic drugs. Optom Clin 1992;2(4):73-96. http://ovidsp.ovid.com/ovidweb.cgi?T=JS\& PAGE=reference $\& D=$ emed $2 \& N E W S=N \& A N=1363080$.

46. Wong J, Lan W, Ong L. Non-hormonal systemic medications and dry eye. Ocul Surf 2010;9(4):1408-11. doi:10.1016/S15420124(11)70034-9.

47. Stapleton F, Marfurt C, Golebiowski B, et al. The TFOS International Workshop on Contact Lens Discomfort : Report of the subcommittee on neurobiology. Invest Ophthalmol Vis Sci 2013;54(11):TFOS71-97. doi:10.1167/iovs.13-13226.

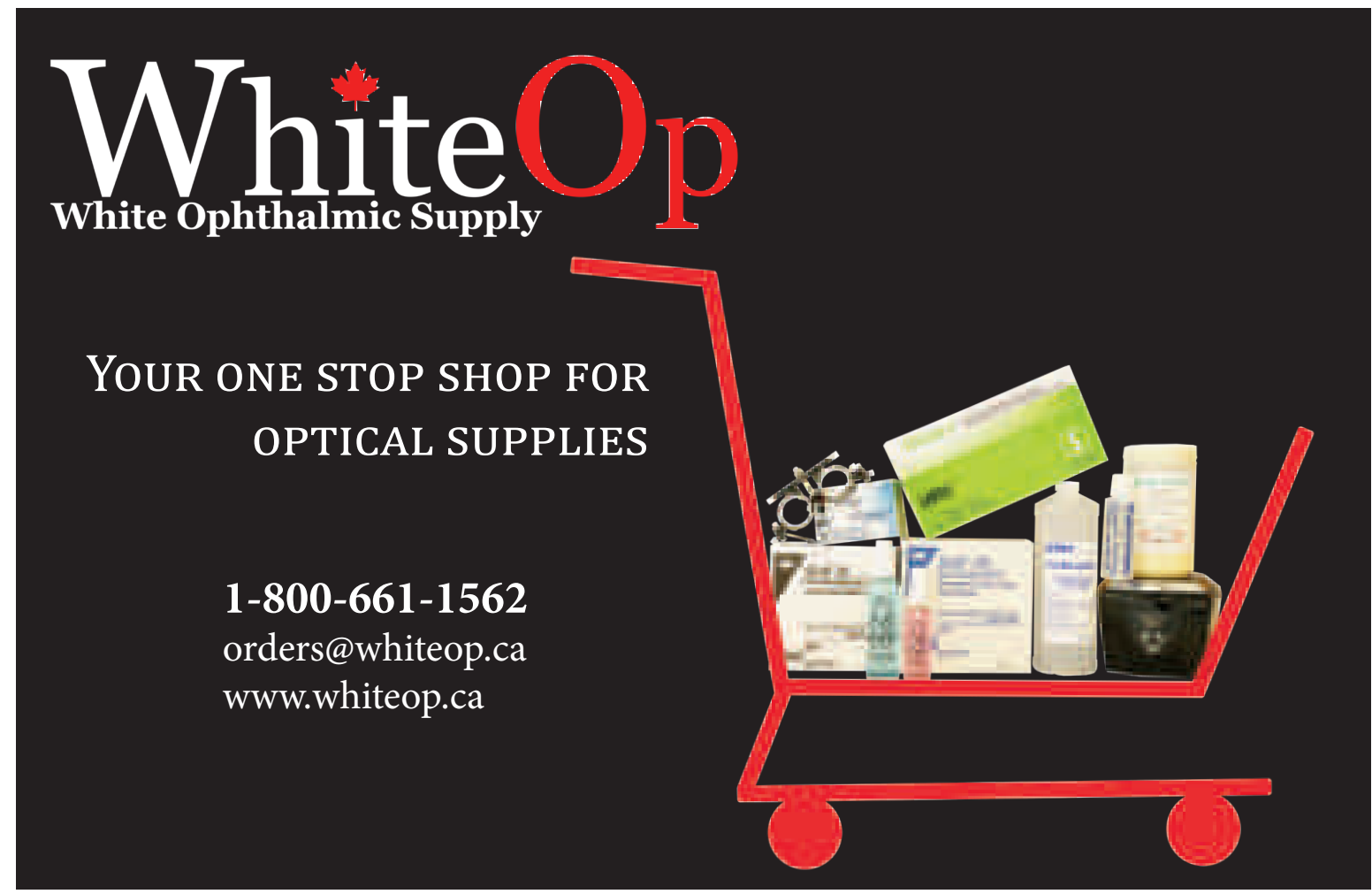

